\title{
Nonlinear Cobweb Model with Time Delays
}

\author{
Akio Matsumoto* \\ Department of Economics \\ Chuo University \\ Tokyo, Japan \\ *Corresponding author
}

\author{
Keiko Nakayama* \\ Department of Economics \\ Chukyou University \\ Nagoya, Japan
}

\begin{abstract}
We study the effects of production delays on the local as well as on global dynamics of nonlinear Cobweb model in a continuous-time frame- work. After reviewing a single delay model, we proceed to two models with two delays. When the two delays are used to form an expected price or feedback for price adjustment, we have a winding stability switching curve and in consequence, obtain repetition of stability losses and gains via Hopf bifurcation.
\end{abstract}

Keywords-continuous time cobweb model; nonlinear price dynamics; production delay; stability switching; hopf bifurcation

\section{INTRODUCTION}

It is now well-known that the cobweb model or cobweb theory has been de- veloped in various directions since the pioneering work of Kaldor (1934). It explains why and how certain types of markets give rise to fluctuations in prices and quantities. Since it mainly focuses on the agricultural markets in which producers determine their outputs before observing market prices and a delay between planting and harvesting is inevitable, its key issues are an expecta- tion formation of price and a production delay. In early stage, the models are essentially linear and constructed in discrete time scales in which production delay is incorporated from the beginning. Thus the main question is on how the expectation formations such as naive, adaptive and rational expectations are responsible for the emergence of fluctuations. During the last two decades, an increasing attention has been given to nonlinear dynamics. The nonlinear and discrete-time cobweb models can generate a wide spectrum of dynamic behavior involving chaos. See Dieci and Westerhof (2010) and Hommes (1994), to name a few

It is, however, less-known that a continuous time cobweb model with fixed time delay is also developed with the same problem consciousness as early as in the 1930s. In particular, Haldane (1933) found the similarity between the effects caused by the rise in the birth rate in biology and the ones by a rise in commodity price in economics and built a simple economic model to examine the fluctuations in price and the rate of production, coaxing the idea from theoretical biology. Independently from Haldane, Larson (1964) presents a linear continuous time model in which a hog cycle is described as a harmonic motion. It is assumed that realized production has 12 month delay from planned production and the rate of production change is proportional to the deviation of price from equilibrium. Mackey (1989) gives a nonlinear price adjustment model with production delay and rigorously derives a stability switching condition for which the stability of equilibrium is lost. Furthermore, it is shown that a Hopf bifurcation takes place and thus the stable equilibrium bifurcates to a limit cycle after the loss of stability. Recently Gori et al. (2014) propose a delay cobweb model with the profit-maximizing behavior to characterize production cycles. Although the delay models have been an object of study for a long time, these are subject to a single delay and little is known about multiple delay models. The purpose of this study is, based on Mackey's formulation, to investigate how multiple delays affect cobweb price dynamics, applying the recent mathematical developments to characterize the stability of two delay differential equations conducted by $\mathrm{Gu}$, et al. (2005) and Lin and Wang (2012)..

\section{BASIC COBWEB MODEL}

We consider price dynamics in a continuous-time framework in which relative variations in market price $p(t)$ is adjusted to be propor- tional to excess demand

$$
\mathrm{dp} / \mathrm{dt}=\mathrm{Kp}(\mathrm{t})[\mathrm{D}(\mathrm{p}(\mathrm{t}))-\mathrm{S}(\mathrm{pe}(\mathrm{t}))]
$$

where $\mathrm{K}>0$ is the adjustment coefficient, pe is the expected price, $\mathrm{D}(\mathrm{p})$ and $\mathrm{S}(\mathrm{pe})$ are the demand and supply functions of commody. Both functions are assumed to be linear,

$$
D(t)=d 1-d 2 p(t) \text { and } S(t)=s 1+s 2 p e(t)
$$

The equilibrium price and quantity satisfy the conditions, $\mathrm{p}^{*}=\mathrm{pe}(\mathrm{t})=\mathrm{p}(\mathrm{t})$ and $\mathrm{q}^{*}=\mathrm{D}\left(\mathrm{p}^{*}\right)=\mathrm{S}\left(\mathrm{p}^{*}\right)$. Substituting the linear functions and taking $\mathrm{pe}(\mathrm{t})=\mathrm{p}(\mathrm{t})$ yield a nonlinear price adjustment equation,

$$
d p(t) / d t=K p(t)[d 1-s 1-(d 2+s 2) p(t))] .
$$

To examine stability of the equilibrium price we linearize the above equation arount $\mathrm{p}=\mathrm{p}^{*}$ and obtain a solution

$$
\mathrm{p}(\mathrm{t})=\mathrm{p}^{*+}+\left[\mathrm{p}(0)-\mathrm{p}^{*}\right] \mathrm{e}-\mathrm{k}(\mathrm{d} 2+\mathrm{s} 2) \mathrm{t}
$$

where $\mathrm{k}=\mathrm{Kp} *$ and $\mathrm{k}(\mathrm{d} 2+\mathrm{s} 2) \mathrm{t}>0$ implies that the equilibrium price is stable.

\section{Cobweb Model with A Single Delay}

A production time delay is introduced into the basic model. Concerning the expectation formation, we start with the simplest form,

Assumption 1. pe $(\mathrm{t})=\mathrm{p}(\mathrm{t}-\tau)$ and $\tau>0$. 
The price adjustment function is now the first-order nonlinear delay differential equation,,

$$
p^{\cdot}(t)=K p(t)[d 1-s 1-d 2 p(t)-s 2 p(t-\tau)]
$$

and a linearized equation in a neighborhood of $\mathrm{p}^{*}$ is

$$
\mathrm{p} \cdot \delta(\mathrm{t})=-\mathrm{kd} 2 \mathrm{p} \delta(\mathrm{t})-\mathrm{ks} 2 \mathrm{p} \delta(\mathrm{t}-\tau)
$$

where $\mathrm{p} \delta(\mathrm{t})=\mathrm{p}(\mathrm{t})-\mathrm{p}^{*}$. Applying the usual method for solving this delay linear equation, we obtain the following result:

Theorem 1(A) If $\mathrm{s} 2 \leq \mathrm{d} 2$, then the equilibrium point is locally asymptotically stable for any positive values of $\tau$ :

Theorem 1(B) If $s 2>d 2$, then there is a threshold value $\tau^{-}$ and the equilibrium point is asymptotically stable if $0<\tau<\tau^{-}$, loses stability as $\tau=\tau^{-}$and bifurcates to a limit cycle if $\tau>\tau^{-}$c

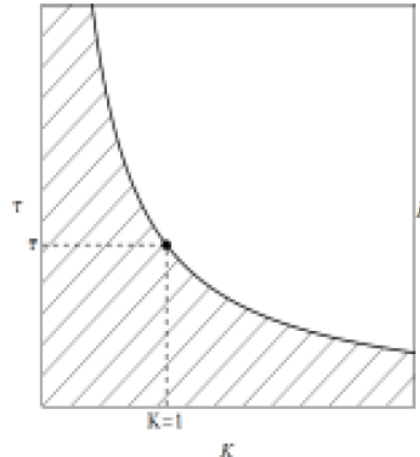

(A) Stability

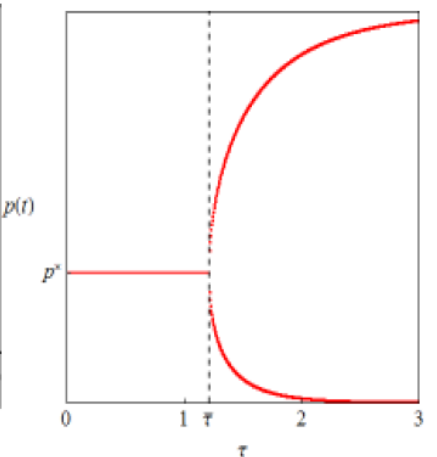

(B) Bifurcation
FIGURE I NUMERICAL RESULTS ON THE ONE DELAY MODEL

\section{CoBweb Model with Two Delays}

The price expectation is formed naïve-wisely under Assumption 1. Convergence to the equilibrium price occurs for $\tau<\tau$. The producers with delay price information can evenetually arrive at the equilibrium price. On the other hand, since cyclic oscillation arises for $\tau>\tau^{-}$, the producers sooner or later realize that their expectations are systematically wrong. As a natural consequence, even though they are assumed to be boundedly rational, the producers may change somehow the way they form expectations. One possible way is to utilize more price information obtained in the past. To make it simpler, they are assumed to use different prices at times, $t-\tau 1$ and $t-$ $\tau 2$. There are at least two different ways to employ these two prices. One is to make the price expectation as the weighted average of these prices. We make the following assumption when the price expectation is the weighted average of the past two prices:

Assumption 2. pe $(\mathrm{t})=\theta \mathrm{p}(\mathrm{t}-\tau 1)+(1-\theta) \mathrm{p}(\mathrm{t}-\tau 2)$ with 0 $<\theta<1$

Substituting this new expectation into the supply function, we obtain the price adjustment governed by a two delay differential equation,

$$
p^{\prime}(t)=K p(t)[(d 1-s 1)-d 2 p(t)-s 2 \theta p(t-\tau 1)-
$$

$$
\mathrm{s} 2(1-\theta) \mathrm{p}(\mathrm{t}-\tau 2)]
$$

The linear approximation in a neighborhood of the equilibrium point is

$$
\mathrm{p} \cdot \delta(\mathrm{t})=-\mathrm{kd} 2 \mathrm{p} \delta(\mathrm{t})-\mathrm{ks} 2 \theta \mathrm{p} \delta(\mathrm{t}-\tau 1)-\mathrm{ks} 2(1-\theta) \mathrm{p} \delta(\mathrm{t}-\tau 2)
$$

which can be reduced to

$$
x^{\cdot}(t)+\alpha x(t)+\beta x(t-\tau 1)+\gamma x(t-\tau 2)=0
$$

with

$$
\mathrm{x}(\mathrm{t})=\mathrm{p} \delta(\mathrm{t}), \alpha=\mathrm{kd} 2, \beta=\mathrm{ks} 2 \theta \text { and } \gamma=\mathrm{ks} 2(1-\theta)
$$

The corresponding characteristic equation is obtained by substituting an exponential solution $x(t)=e^{\lambda t} u$

$$
\lambda+\mathrm{a}+\beta \mathrm{e}-\lambda \tau 1+\gamma \mathrm{e}-\lambda \tau 2=0
$$

Stability depends on the Stability depends on the locations of the eigenvalues of this equation that is investigated by applying the method developed by $\mathrm{Gu}$ et al (2005).Dividing both side of the characteristic equation by $\lambda+a$ and introducing the new functions,

$$
\mathrm{a} 1(\lambda)=\beta /(\lambda+\mathrm{a}) \text { and } \mathrm{a} 2(\lambda)=\beta \omega /(\lambda+\mathrm{a})
$$

simplify the left hand side of the characteristic equation,

$$
\mathrm{a}(\lambda)=1+\mathrm{a} 1(\lambda) \mathrm{e}-\lambda \tau 1+\mathrm{a} 2(\lambda) \mathrm{e}-\lambda \tau 2
$$

Substituting a possible solution $\lambda=i \omega$ with $\omega>0$ into the two equations above results in

$$
a 1(i \omega)=a \beta /(a 2+\omega 2)-i \beta \omega /(a 2+\omega 2)
$$

and

$$
\mathrm{a} 2(\mathrm{i} \omega)=\mathrm{a} \gamma /(\mathrm{a} 2+\omega 2)-i \gamma \omega /(\mathrm{a} 2+\omega 2)
$$

Their absolute values are

$$
|\mathrm{a} 1(\mathrm{i} \omega)|=\beta /(\mathrm{a} 2+\omega 2) 1 / 2 \text { and }|\mathrm{a} 2(\mathrm{i} \omega)|=\gamma /(\mathrm{a} 2+\omega 2) 1 / 2
$$

We can consider the three terms in $\mathrm{a}(\lambda)$ as three vectors in the complex plane with the magnitudes, $1,|\mathrm{a} 1(\lambda)|$ and $|\mathrm{a} 2(\lambda)|$. The solution $\mathrm{a}(\lambda)=0$ means that these vectors form a triangle if we put them head to tail. That is, solving $a(\lambda)=0$ algebratically is equivalent to constructing a triangle geometrically with the following three conditions,

$$
\begin{aligned}
& 1 \leq|\mathrm{a} 1(\mathrm{i} \omega)|+|\mathrm{a} 2(\mathrm{i} \omega)| \\
& |\mathrm{a} 1(\mathrm{i} \omega)| \leq 1+|\mathrm{a} 2(\mathrm{i} \omega)|
\end{aligned}
$$

And

$$
|\mathrm{a} 2(\mathrm{i} \omega)| \leq 1+|\mathrm{a} 1(\mathrm{i} \omega)|
$$


Substituting the absolute values converts these three conditions to the following two conditions,

$$
(\beta-\gamma) 2-\alpha 2 \leq \omega 2 \leq(\beta+\gamma) 2-\alpha 2
$$

That can be rewritten as

$$
\mathrm{k} 2[\mathrm{~s} 22(2 \theta-1) 2-\mathrm{d} 22] \leq \omega 2 \leq \mathrm{k} 2(\mathrm{~s} 22-\mathrm{d} 22) .
$$

It is clear that the second inequality does not hold if $\mathrm{d} 2 \geq \mathrm{s} 2$. hHence there is no $\omega>0$,implying that the delays are harmless in the two delay dynamic model.

Theorems 2. If $s_{2} \leq d_{2}$, then the equilibrium is locally asymptotically stable for any positive values of $\tau_{1}$ and $\tau_{2}$.

On the other hand, the conditions hold for $\omega$ in the interrval $\left[\omega_{s}, \omega_{e}\right]$ where

$$
\omega \mathrm{s},=0 \text { if } \theta \leq \theta 0
$$

and

$$
\omega \mathrm{s}=\mathrm{k}(\mathrm{s} 22(2 \theta-1) 2-\mathrm{d} 22) 1 / 2 \text { if } \theta>\theta 0
$$

with

$$
\theta 0=(1+\mathrm{d} 2 / \mathrm{s} 2) / 2
$$

that solves $s_{2}^{2}(2 \theta-1)^{2}-d_{2}^{2}=0$ and

$$
\omega \mathrm{e}=\mathrm{k}(\mathrm{s} 22-\mathrm{d} 22) 1 / 2
$$

We will next find the pair of $(\tau 1, \tau 2)$ satisfying a $(i \omega)=0$. Let $|1|$ be the base of the triangle and then dnote an angle between $|1|$ and $|\mathrm{a} 1(\mathrm{i} \omega)|$ by $\theta 1$ and $|1|$ and $|\mathrm{a} 2(\mathrm{i} \omega)|$ by $\theta 2$. By the law of cosine, the angle's magnitude are expressed in terms of the model parameters,

$$
\left.\theta 1(\omega)=\cos ^{-1} 1(\omega 2+\alpha 2+\beta 2-\gamma 2) /(2 \beta(\alpha 2+\omega 2) 1 / 2)\right]
$$

and

$$
\theta 2(\omega)=\cos ^{-1} 1[(\omega 2+\alpha 2-\beta 2+\gamma 2) /(2 \gamma(\alpha 2+\omega 2) 1 / 2)]
$$

We can obtain the arguments,

$$
\begin{aligned}
& \arg [\operatorname{ar}(i \omega)]=-\tan -1(\omega / \alpha) \\
& \arg [\operatorname{a} 2(i \omega)]=-\tan -1(\omega / \alpha)
\end{aligned}
$$

Since the triangle can be located above and under the real axis, the following two equations hold for $\tau 1$ and $\tau 2$ :

$$
\begin{aligned}
& \{\arg [\operatorname{ar}(\mathrm{i} \omega) \mathrm{e}-\mathrm{i} \omega \tau 1]+2 \mathrm{~m} \pi\} \pm \theta 1(\omega)=\pi \\
& \{\arg [\mathrm{a} 2(\mathrm{i} \omega) \mathrm{e}-\mathrm{i} \omega \tau 2]+2 \mathrm{n} \pi\} \mp \theta 2(\omega)=\pi
\end{aligned}
$$

Which yield the threshold values of the delays

$$
\begin{gathered}
\tau 1 \pm(\omega, \mathrm{m})=[-\tan -1(\omega / \alpha)+(2 \mathrm{~m}-1) \pi \pm \theta 1(\omega)] / \omega \\
\tau 2 \mp \omega, \mathrm{m})=[-\tan -1(\omega / \alpha)+(2 \mathrm{n}-1) \pi \mp \theta 2(\omega)] / \omega
\end{gathered}
$$

Here $m$ and $n$ are nonnegative integers such that $\tau 1>0$ and $\tau 2>0$. Thus for any $\mathrm{m}, \mathrm{n}$ and $\omega \in[\omega \mathrm{s}, \omega \mathrm{e}]$, we can define the pair of $(\tau 1, \tau 2)$ constructing the stability switching curve as follows:

Theorem 4 Given $\mathrm{s} 2>\mathrm{d} 2$, the stability switching curve is described by $\mathrm{C} 1(\mathrm{~m}, \mathrm{n}) \cup \mathrm{C} 2(\mathrm{~m}, \mathrm{n})$ with $\mathrm{m}, \mathrm{n}=0,1,2 \ldots$, where

$$
\mathrm{C} 1(\mathrm{~m}, \mathrm{n})=\{\tau 1+(\omega, \mathrm{m}), \tau 2-(\omega, \mathrm{n})\}
$$

and

$$
\mathrm{C} 2(\mathrm{~m}, \mathrm{n})=\{\tau 1-(\omega, \mathrm{m}), \tau 2+(\omega, \mathrm{n})\}
$$

The segments $\mathrm{C} 1(\mathrm{~m}, \mathrm{n}+1)$ and $\mathrm{C} 2(\mathrm{~m}, \mathrm{n})$ have the same starting point where as the segments $\mathrm{C} 1(\mathrm{~m}, \mathrm{n})$ and $\mathrm{C} 2(\mathrm{~m}, \mathrm{n})$ have the same end point.

Under Assumption 2 with $\theta=0.8$ and $\mathrm{m}=1$, the stability switching curve is illustrated in Figure II in which the red segments show $\mathrm{C} 1(1, \mathrm{n})$ and the blue segments show $\mathrm{C} 2(1, \mathrm{n})$ for $n=1,2,3$. Notice the segment shifts upward as the value of $\mathrm{n}$ increases and to the right as the value of $\mathrm{m}$ increases. The lowest red and blue segments are $\mathrm{C} 1(1,1)$ and $\mathrm{C} 2(1,1)$ where they are connected to each other at point $\mathrm{C} 1 \mathrm{e}(1,1)=\mathrm{C} 2 \mathrm{e}(1,1)$. The middle red and blue segments are

$\mathrm{C} 1(1,2)$ and $\mathrm{C} 2(1,2)$ where $\mathrm{C} 1(1,2)$ is connected to $\mathrm{C} 2(1$, 1) at $\mathrm{C} 1 \mathrm{~s}(1,2)=\mathrm{C} 2 \mathrm{~s}(1,1)$ As $\mathrm{n}$ increases the two segments are connected in the same way to construct the continuous stability switching curve, $\mathrm{C} 1(1, \mathrm{n}) \cup \mathrm{C} 2(1, \mathrm{n})$. This curve divides the first quadrant into two parts as shown in Figure II. One contains the origin

And its every point can be reached from the origin via continuous curve not crossing the stability switching curve. At any point in this region, the real parts of the eigenvalues are negative, so the system is locally asymptotically stable. On the other hand, at the points in the complement of this region except the stability switching curve, the system is unstable. Observing Figure II, we find the following three issues:

(1) For $\tau 1 \leq \tau 1 \mathrm{~h} \simeq 1.188$, the system is locally asymptotically stable irrespective of the values of $\tau 2$, implying that delay $\tau 2$ is harmless;

(2) For $\tau 1>\tau 1 \mathrm{~h}$, stability loss and gain repeatedly occur when $\tau 2$ increases from zero;

(3) Depending on the value of $\tau 2$, two different dynamic phenomena are seen when the value of $\tau 1$ increases. One is when stability is lost and cannot be regained as in the one delay model, and the other case is when stability regain can occur. 


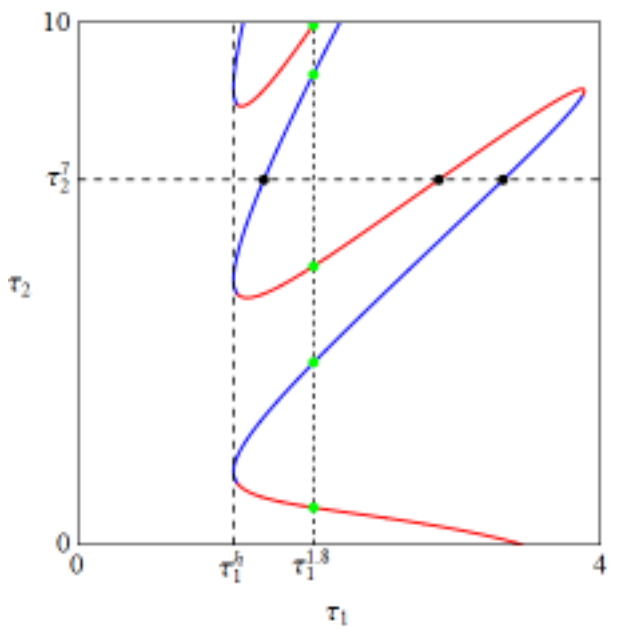

FIGURE II STABILITY SWITCHING CURVE

We now examine the effect caused by changing $\tau 2$, keeping the value of $\tau 1$ at some positive value. In Figure III(A) the bifurcation diagram of $\mathrm{p}(\mathrm{t})$ with respect to $\tau 2$ is illustrated. The value of $\tau 1$ is fixed at $\tau 1.8=1.8$ and the value of $\tau 2$ is increased along the vertical dotted line at $\tau 1.8$ in Figure II. For each value of $\tau 2$, the dynamic system runs for $0 \leq t \leq 1000$ and discard the data for $0 \leq \mathrm{t} \leq 950$ to get rid of the transients. The local maximum and minimum obtained from the remaining data are plotted against the value of $\tau 2$. The valu e of $\tau 2$ is increased with $1 / 400$ and then the same procedure is repeated until $\tau 2$ arrives at 10 . If the resultant bifurcation diagram has only one point against the value of $\tau 2$, then the system is locally stable and that point corresponds to the stationary point. If it has two points, then a limit cycle with one maximum and one minimum emerges. As is seen in Figure II, the vertical line at $\tau 1=\tau 1.8$ a crosses the stability switching curves five times. We denote the values of $\tau 2$ of the green intersection points $\tau 2$ $\mathrm{a}, \tau 2 \mathrm{~b}, \tau 2 \mathrm{c}, \tau 2 \mathrm{~d}$ and $\tau 2 \mathrm{e}$ in the ascending order. The bifurcation diagram in Figure III(A) indicates the following dynamics: after stability is lost at $\tau 2$ a, a limit cycle emerges and its amplitude firs expand, then shrinks to zero at $\tau=\tau 2 \mathrm{~b}$ when stability is regained. The same process is repeated for larger values of $\tau 2$.

We draw attention to the effect caused by changing the value of $\tau 1$. With the similar procedure, Figure III (B) illustrates the bifurcation diagram with respect to $\tau 1$ along the dotted horizontal line at $\tau 2=\tau 7(=7)$ shown in Figure II in which the line crosses the stability switching line three times denoted by three black dots at $\tau \mathrm{A}(\simeq 1.421), \tau \mathrm{B}(\simeq 2.758)$ and $\tau \mathrm{C}(\simeq 3.252)$ in the ascending order. A limit cycle emerges when stability lost and stability losses and gains are repeatedly observed.
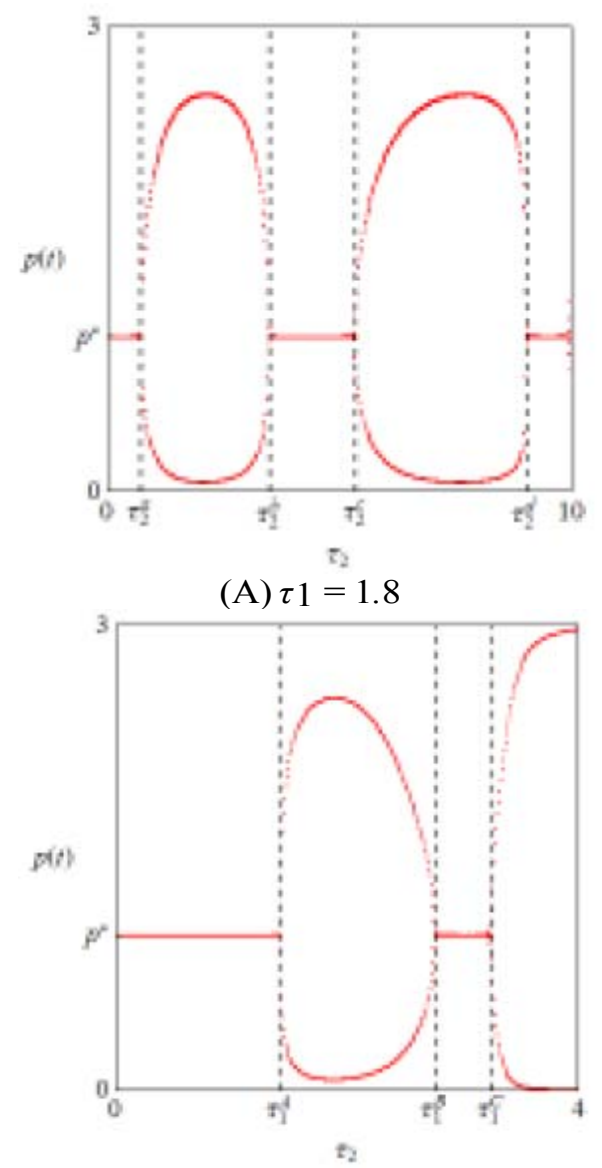

(B) $\tau 2=7$

FIGURE III BIFURCATION DIAGRAM

\section{CONCLUDING REMARKS}

In this study, we have examined the delay effect on price dynamics in three different models. After reviewing a single delay model in which a limit cycle can emerge via Hopf bifurcation, we proceed to two models with two production delays. When the two delays are used to form an expected price or feedback for price adjustment, we find that the stability switching curve on which stability is changed is winding and as a natural consequence, stability losses and gains are repeated when the length of one delay increases. It is numerically confirmed that only simple dynamics such as a limit cycle can emerge when stability is lost.

\section{ACKNOWLEDGMENT}

The authors highly appreciate the financial supports from the MEXT-Supported Program for the Strategic Research Foundation at Private Universities 2013-207, the Japan Society for the Promotion of Science (Grant-in-Aid for Scientific Research (C) 24530202, 25380238 and 26380316). The usual disclaimers apply.

\section{REFERENCES}

[1] Dieci, R. and Westerhof, F. Interacting Cobweb Markets, Journal of Eco- nomic Behavior and Organization, 75, 461-181, 2010

[2] Ezekiel, M. The Cobweb Theorem, Quarterly Journal of Economics, 52, 255-280, 1938. 
[3] Gori, L., Guerrini, L. and Sodini, M. Hopf Bifurcation in a Cobweb Model with Discrete Time Delays, Discrete Dynamics in Nature and Society, $2014, \quad$ Article $\quad$ ID $\quad 137090$ (http://dx.doi.org/10.1155/2014/137090), 8 pages, 2014..

[4] Gu, K., Niculescu, S. and Chen, J. On Stability Crossing Curves for General Systems with Two Delays, Journal of Mathematical Analysis and Applica- tions, 311, 231-252, 2005

[5] Kaldor, N. A Classificatory Note on the Determinateness of Equilibrium,Review of Economic Studies, 1, 122-136, 1934.Hommes, C. Dynamics of the Cobweb Model with Adaptive Expectations and Nonlinear Supply and Demand, Journal of Economic Behavior and Organization, 24, 315-335, 1994

[6] Haldane, J. A Contribution to the Theory of Price Fluctuations, Review of Economic Studies, 1, 186-195, 1933.

[7] Hommes, C. Dynamics of the Cobweb Model with Adaptive Expectations and Nonlinear Supply and Demand, Journal of Economic Behavior and Organization, 24, 315-335, 1994.

[8] Larson, A. The Hog Cycle as Harmonic Motion, Journal of Farm Economics, 46, 375-386, 1964.

[9] Lin, X. and Wang, H. Stability Analysis of Delay Differential Equations with Two Discrete Time Delays, Canadian Applied Mathematics Quarterly, 20, 519-533, 2012.

[10] Mackey, M. Commodity Price Fluctuations: Price Dependent Delays and Nonlinearities as Explanatory Factors, Journal of Economic Theory, 48, 497-509, 1989.

[11] Matsumoto, A. and Szidarovszky, F. Nonlinear Cournot Duopoly with Im- plementation Delays, IERCU Discussion Paper \#240, Institute of Econoic Research, Chuo University, 2 